\title{
Progranulin Deficiency Decreases Gross Neural Connectivity But Enhances Transmission at Individual Synapses
}

\author{
Lucia Tapia, ${ }^{1,5}$ Austen Milnerwood, ${ }^{2,5}$ Aobo Guo, ${ }^{3,5}$ Fergil Mills, ${ }^{1,5}$ Eileen Yoshida, ${ }^{1,5}$ Cristina Vasuta, ${ }^{2,5}$ \\ Ian R. Mackenzie, , ${ }^{5,6}$ Lynn Raymond, ${ }^{2,5}$ Max Cynader, ${ }^{4,5}$ William Jia, ${ }^{3,5}$ and Shernaz X. Bamji ${ }^{1,5}$ \\ Departments of ${ }^{1}$ Cellular and Physiological Sciences and ${ }^{2}$ Psychiatry, University of British Columbia, Vancouver, British Columbia V6T 1Z3, Canada, \\ Departments of ${ }^{3}$ Neurosurgery and ${ }^{4}$ Ophthalmology and ${ }^{5}$ Brain Research Centre, University of British Columbia, Vancouver, British Columbia V6T 2B5, \\ Canada, and ${ }^{6}$ Department of Pathology and Laboratory Medicine, Vancouver General Hospital, Vancouver, British Columbia V5Z 2G9, Canada
}

Frontotemporal dementia (FTD) has been linked to mutations in the progranulin gene (GRN) that lead to progranulin (PGRN) haploinsufficiency. Thus far, our understanding of the effects of PGRN depletion in the brain has been derived from investigation of gross pathology, and more detailed analyses of cellular function have been lacking. We report that knocking down PGRN levels in rat primary hippocampal cultures reduces neural connectivity by decreasing neuronal arborization and length as well as synapse density. Despite this, the number of synaptic vesicles per synapse and the frequency of mEPSCs are increased in PGRN knockdown cells, suggesting an increase in the probability of release at remaining synapses. Interestingly, we demonstrate that the number of vesicles per synapse is also increased in postmortem brain sections from FTD patients with PGRN haploinsufficiency, relative to controls. Our observations show that PGRN knockdown severely alters neuronal connectivity in vitro and that the synaptic vesicle phenotype observed in culture is consistent with that observed in the hippocampus of FTD patients.

\section{Introduction}

Progranulin (PGRN) is a multifunctional secreted growth factor that is widely expressed in the developing brain but becomes progressively restricted to defined neuronal populations, including cortical and hippocampal pyramidal neurons and Purkinje cells (Daniel et al., 2000, 2003; Petkau et al., 2010). Thus far, PGRN has been implicated in the regulation of neuronal survival (Van Damme et al., 2008; Ryan et al., 2009; Kleinberger et al., 2010), and treatment of neurons with exogenous PGRN has been shown to enhance neurite outgrowth (Van Damme et al., 2008; Gao et al., 2010). Despite this, an understanding of the role of PGRN in the development and maintenance of neural circuitry is still in its infancy.

Interest in understanding the role of PGRN in the brain has been heightened following the discovery that mutations in the progranulin gene (GRN) are a major cause of autosomal dominant, tau-negative frontotemporal dementia (FTD) (Baker et al., 2006; Cruts et al., 2006; Gass et al., 2006; Mukherjee et al., 2006; Pickering-Brown et al., 2006; Bronner et al., 2007; van der Zee et

Received Nov. 30, 2010; revised June 7, 2011; accepted June 13, 2011.

Author contributions: L.T., L.R., M.C., W.J., and S.X.B. designed research; L.T., A.M., A.G., F.M., E.Y., and C.V. performed research; I.R.M. and W.J. contributed unpublished reagents/analytic tools; L.T., A.M., F.M., and E.Y. analyzed data; L.T. and S.X.B. wrote the paper.

This work was supported by grants from Canadian Institutes of Health Research (CIHR MOP-81158 to S.X.B., CIHR MOP-12699 to L.R.) and the Pacific Alzheimer's Research Foundation to W.J. We thank Dr. Jamie Boyd for technical assistance.

Correspondence should be addressed to either of the following: Dr. ShernazX. Bamji, Department of Cellular and Physiological Sciences, University of British Columbia, 2350 Health Sciences Mall, Vancouver, BC V6T 1Z3, Canada, E-mail: shernaz.bamji@ubc.ca; or Dr. William Jia, Department of Neurosurgery, University of British Columbia, 2211 Wesbrook Mall, M42A, Purdy Pavilion, Vancouver, BC V6T 2B5, Canada, E-mail: wjia@interchange.ubc.ca.

DOI:10.1523/JNEUROSCI.6244-10.2011

Copyright $\odot 2011$ the authors $\quad 0270-6474 / 11 / 3111126-07 \$ 15.00 / 0$ al., 2007). The majority of GRN mutations produce null alleles, indicating that FTD results from a loss of functional PGRN (haploinsufficiency) (Baker et al., 2006; Gass et al., 2006). PGRN pathology has also been reported in other dementia-related and neurodegenerative diseases (Malaspina et al., 2001; Baker and Manuelidis, 2003; Baker et al., 2006). Thus, it is imperative to improve our understanding of PGRN function in the brain, and to determine how alterations in PGRN levels impact basic neural circuitry.

\section{Materials and Methods}

Neuron cultures

Hippocampal neuronal cultures were prepared from E18 rat embryos as previously described (Xie et al., 2000). Neurons were infected at $3 \mathrm{~d}$ in vitro (DIV) with a lentivirus expressing GFP plus either a scrambled siRNA (control) or PGRN siRNA. For rescue experiments, cells were reinfected at 8 DIV with empty vector $(+\mathrm{V})$, or a vector expressing a siRNA-insensitive, human PGRN $(+\mathrm{P})$. For analysis of neuronal morphology and synapse density, 11 DIV neurons were transfected with DsRed or PSD-95-RFP (kind gift from Dr. David Bredt, Indianapolis, IN), respectively, using Lipofectamine 2000 (Invitrogen). Analyses were done at 13 DIV.

siRNA constructs, lentivirus infection, and recombinant DNAs PGRN siRNA (5'-GACAGAGTGCATTGCTGTC-3') and scrambled siRNA (5'-GCCTATGGTCACAGGTAGT-3') oligonucleotides were generated (Integrated DNA Technologies) and inserted into pSUPER. The lentivirus also expressed GFP under the synapsin promoter. A multiplicity of infection of 5 was used in all cases. siRNA-insensitive, human PGRN (NM_002087.2) (gift from Dr. Andrew Bateman, Montreal, Canada) was inserted in an FUW lentivirus vector at BamHI and EcoRI sites.

Immunohistochemistry

Cultures were immunolabeled as previously described (Sun et al., 2009).

Primary antibodies were as follows: rabbit anti-synaptophysin (Sigma), 
A

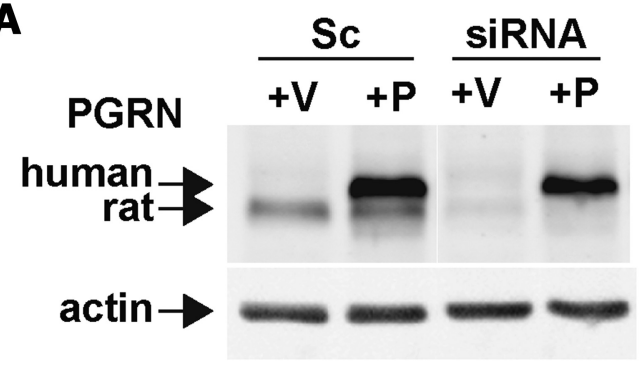

B

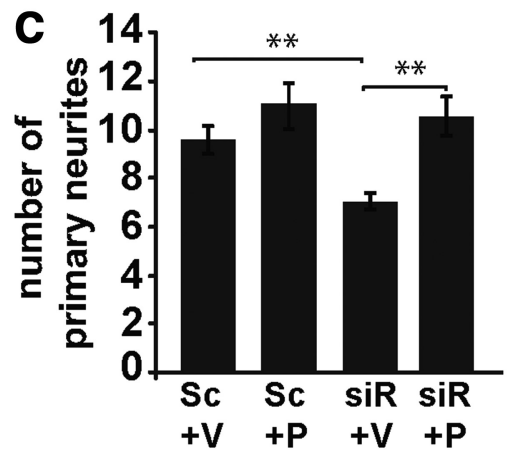

E
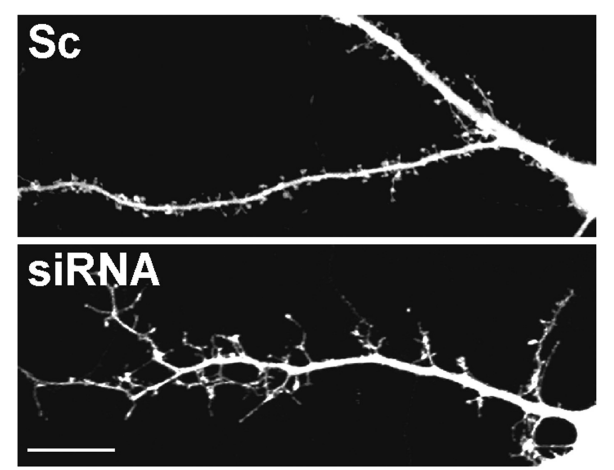

D

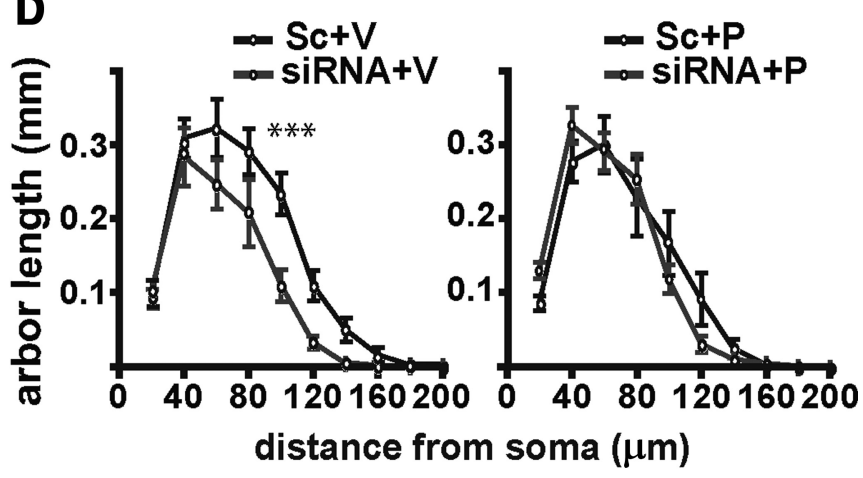

$\mathbf{F}$

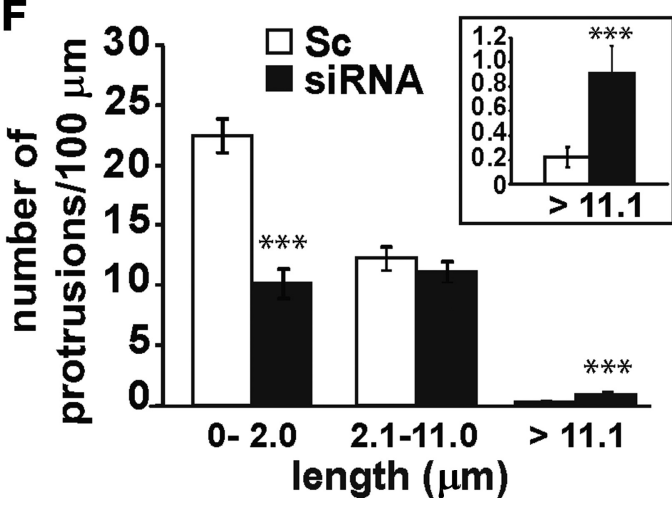

Figure 1. Dendritic arborization and spine density are reduced in PGRN-deficient neurons. $A$, Representative Western blot from hippocampal cultures infected with scrambled (Sc) or PGRN siRNA (siRNA), and subsequently infected with empty vector $(+V)$, or a vector expressing human PGRN $(+P)$. $\boldsymbol{B}, \boldsymbol{E}$, Confocal images of hippocampal neurons infected with scrambled or PGRN siRNA and transiently transfected with dsRed. $\boldsymbol{C}, \boldsymbol{D}, \boldsymbol{F}$, The number of primary neurites $(\boldsymbol{C}$, arbor length $(\boldsymbol{D})$, and the density of dendritic protrusions $(\boldsymbol{F})$ are significantly decreased in cells expressing PGRN siRNA relative to controls. Subsequent expression of PGRN eliminated differences between $S$ c and siRNA transfected cells $(\boldsymbol{C}, \boldsymbol{D})$. $\boldsymbol{F}$, Histogram of the density of dendritic protrusions. $n=6$ to 20 cells per condition from 5-8 separate cultures. ${ }^{* *} p<0.01,{ }^{* * *} p<0.001$ for one-way ANOVA with Tukey post hoc $(\boldsymbol{C})$ and for Student's $t$ test $(\boldsymbol{F}) . \boldsymbol{D},{ }^{* * *} p<0.0001, F_{(1,319)}=18.3$, two-way ANOVA, treatment. Scale bar, $10 \mu \mathrm{m}$.

mouse anti-PSD-95 (Affinity BioReagents), and guinea pig anti-VGAT and anti-VGluT-1 (Synaptic Systems). Secondary antibodies were as follows: Alexa 488, Alexa 633, and Texas Red-conjugated goat anti-mouse or anti-rabbit (Invitrogen).

\section{TUNEL and MTT assays}

MTT assays were done as described by Korting et al. (1994). $n=3$ independent cultures; 4 wells per condition per culture. TUNEL assays were conducted with the NeuroTACSII In Situ Apoptosis Detection Kit (R\&D Systems). Approximately 1300 cells were counted per experiment. $n=4$ independent cultures; 10 images per condition per culture.

\section{FM4-64 labeling}

FM4-64 experiments were done as previously described (Sun et al., 2009). ADVESAP-7 (1 mm; Sigma) was added to quench nonspecific signal. Three images were captured to confirm that the positive sites of FM loading were stationary presynaptic terminals. Unloading was done for $30 \mathrm{~s}$ in the same hyperkalemic solution used to load the FM4-64 dye. Unloading of FM4-64 to 15\% of initial intensity was observed in $>95 \%$ of the labeled sites.
Immunoblot analysis

Cell lysates were prepared as previously described (Sun et al., 2009). Antibodies were as follows: rabbit anti-synaptophysin (Sigma) and mouse anti-progranulin (R\&D Systems), anti-GluR2 (Millipore Bioscience Research Reagents), and anti-actin (Sigma). Biotinylation and lysis of cultures were done as per Holman and Henley (2007).

\section{Electrophysiology}

General recording conditions were as previously described (Milnerwood and Raymond, 2007). Cells were perfused (2-4 ml/min) with the following (in mM): $167 \mathrm{NaCl}, 2.4 \mathrm{KCl}, 10$ glucose, $10 \mathrm{HEPES}, 2 \mathrm{CaCl}_{2}$, and 1 $\mathrm{MgCl}_{2}, \mathrm{pH} 7.4,300-305 \mathrm{mOsm} / \mathrm{L}$ ) at $27-30^{\circ} \mathrm{C}$. Whole-cell patch-clamp recordings of miniature EPSCs (mEPSCs) isolated by $100 \mu \mathrm{m}$ picrotoxin and $200 \mathrm{~nm}$ tetrodotoxin were obtained. Pipette resistance was 3-6 M $\Omega$ when filled with (in mM) 130 Cs methanesulfonate, $5 \mathrm{CsCl}, 4 \mathrm{NaCl}, 1$ $\mathrm{MgCl}_{2}, 5$ EGTA, 10 HEPES, 5 QX-314, 0.5 GTP, $10 \mathrm{Na}_{2}-$ phosphocreatine, and $5 \mathrm{MgATP}, \mathrm{pH} 7.4,290-295 \mathrm{mOsm} / \mathrm{L}$. Series resistances $\left(R_{\mathrm{s}} ;<20 \mathrm{M} \Omega\right)$ were uncompensated and did not differ between groups (mean $11.8 \pm 0.6 \mathrm{M} \Omega$ ); cells were discarded if $R_{\mathrm{s}}$ changed $>10 \%$ or if $I_{\mathrm{h}}$ exceeded $-180 \mathrm{pA}$ (mean $-43.4 \pm 6 \mathrm{pA} \mathrm{M} \Omega$ ). mEPSCs were 
isolated using Clampfit10 (detection threshold 8 pA; typically $2-3$ rms noise), and nonunitary events were suppressed from all analyses other than frequency and interevent intervals. Statistics were performed with Prism 6 (GraphPad Software) software and significance determined as $p<0.05$.

\section{PGRN genetic analysis}

Three FTD samples were used: (1) UBC-11; male; age of onset: 68 ; age at death: 76 ; severity out of 4,4 ; (2) UBC-17; male; age of onset: 58 ; age at death: 61 ; severity out of 4,3 ; (3) UBC-15; female; age of onset: 69; age at death: 77; severity out of 4,4 . For all cases, tissue samples were fixed $<24 \mathrm{~h}$ postmortem. Additional information has been published by Baker et al. (2006).

\section{Electron microscopy}

Postmortem sections. We embedded $1 \mathrm{~mm} \times 2$ $\mathrm{mm}$ sections of hippocampi in a JEMBED/ Spurr's mixture and stained $70 \mathrm{~nm}$ sections with $2 \%$ uranyl acetate and Reynold's lead. Over 50 images from the stratum radiatum directly underlying the CA1 stratum pyramidale were analyzed for each patient. Images were acquired at $60,000 \times$ with experimenter blind to genotype.

Hippocampal cultures. Hippocampal cultures were plated on polyester membrane inserts in a multiwell chamber (Costar) previously treated with poly-L-lysine. At 13 DIV, membranes were cut with a biopsy cutter and processed as above. Over 50 separate images (magnification 70,000-100,000×) were acquired for each treatment and analyzed with the experimenter blind to the experimental condition.

\section{Image analysis and quantification}

Hippocampal neurons were imaged using an Olympus Fluoview 1000 confocal microscope $(60 \times / 1.4$ Oil Plan-Apochromat). All images in a given experiment were captured and analyzed with the same exposure time and conditions.

Neuronal morphology. Individual cells were transfected with DsRed. The number of primary dendrites and the number and length of spiny protrusions were analyzed using ImageJ. To determine neurite complexity and lengths by Sholl analysis, neurite lengths in $20 \mu \mathrm{m}$ increments from the soma were quantified using a custom software routine in Igor Pro (WaveMetrics) designed in house as used by Milnerwood et al. (2010). To determine the density and length of spiny protrusions, all protrusions along primary dendrites within $100 \mu \mathrm{m}$ from the cell body were analyzed.

Synapse density. Cultures transfected with PSD-95-RFP were fixed and immunostained with anti-synaptophysin. Images were analyzed using ImageJ with a colocalization plugin (http:// rsb.info.nih.gov/ij/plugins/ colocalization.html). Points of colocalization were defined as regions $>1$ pixel in size where the intensity ratio of the two channels was $>50$.

Punctum number and size. Puncta for FM4-64, synaptophysin, PSD95, VGluT-1, and VGAT were analyzed using ImageJ. Over 30 images from different fields were taken for each group using the same settings within groups. The threshold values were chosen based on a subjective evaluation of "real" clusters compared to background noise as described by Sun et al. (2009). Once the threshold was set, it was applied throughout. All puncta in a field were analyzed. with Tukey post hoc $(\boldsymbol{A}, \boldsymbol{C}, \boldsymbol{G})$.
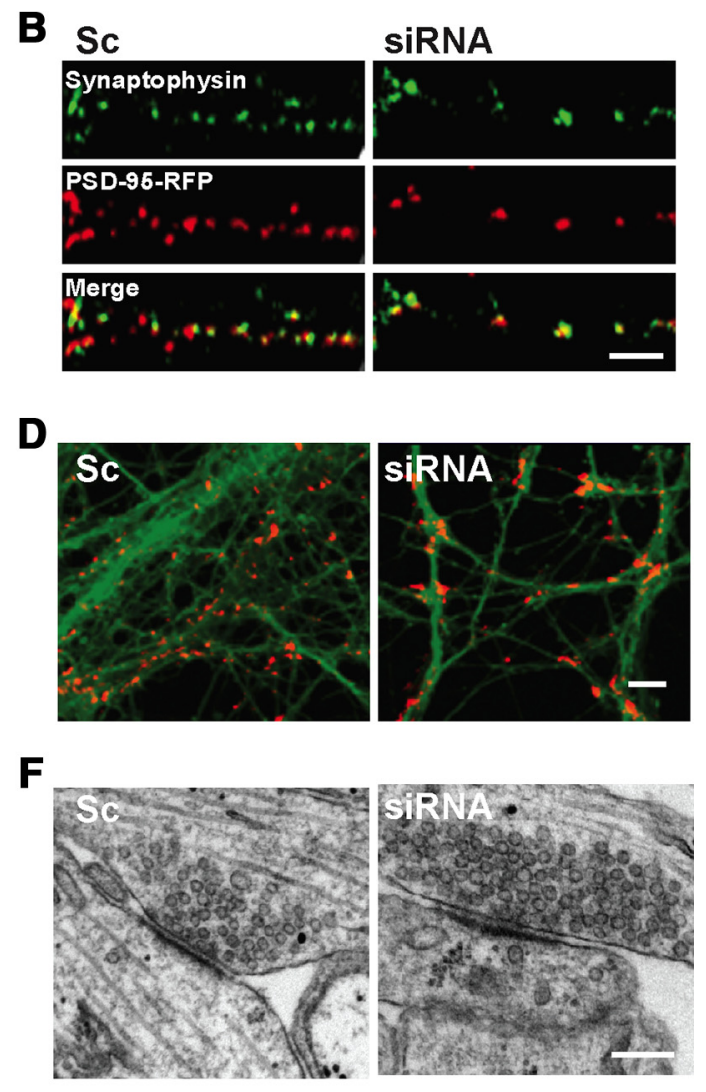

Figure 2. The density of presynaptic clusters is reduced but the number of synaptic vesicles per synapse is increased in PGRNdeficient neurons. $\boldsymbol{A}, \boldsymbol{B}$, The density of putative synapses defined by colocalization of presynaptic and postsynaptic markers is decreased in PGRN knockdown cultures ( $n=9-18$ neurons per condition, 5 cultures). $\boldsymbol{B}$, Confocal images of hippocampal neurons infected with scrambled (Sc) or PGRN siRNA, transiently transfected with PSD-95-RFP, and immunostained for synaptophysin. ures. D, Confocal images of neurons infected with scrambled or PGRN siRNA (green) and fixed and immunolabeled with cale bar, $5 \mu \mathrm{m}$. $\boldsymbol{E}$, Overall synaptophysin levels are unchanged in contro and PGRN siRNAcultures compared to control ( $n=41-52$ synapses per condition, 3 cultures). Scale bar, $100 \mathrm{~nm}$. ${ }^{* *} p<0.001$, one-way ANOVA

Number of synaptic vesicles per synapse (electron microscopy). Synapses in electron micrographs were identified by an electron-dense region associated with at least five vesicles presynaptically, and apposed to an electron-dense postsynaptic density. Only vesicles within $550 \mathrm{~nm}$ from the presynaptic active zone were counted.

Statistical analysis. Significance was determined using a two-tailed Student's $t$ test unless otherwise specified.

\section{Results}

The neuropathology observed in late-stage FTD patients includes a marked compromise in the survival of hippocampal pyramidal neurons. However, it is unclear how GRN deficiency might impact this population of neurons before cell death. To examine this, we knocked down PGRN in cultured primary hippocampal cultures by infection with a lentivirus expressing GFP plus either 
A

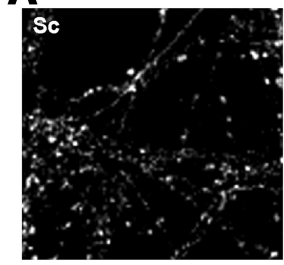
SIRNA
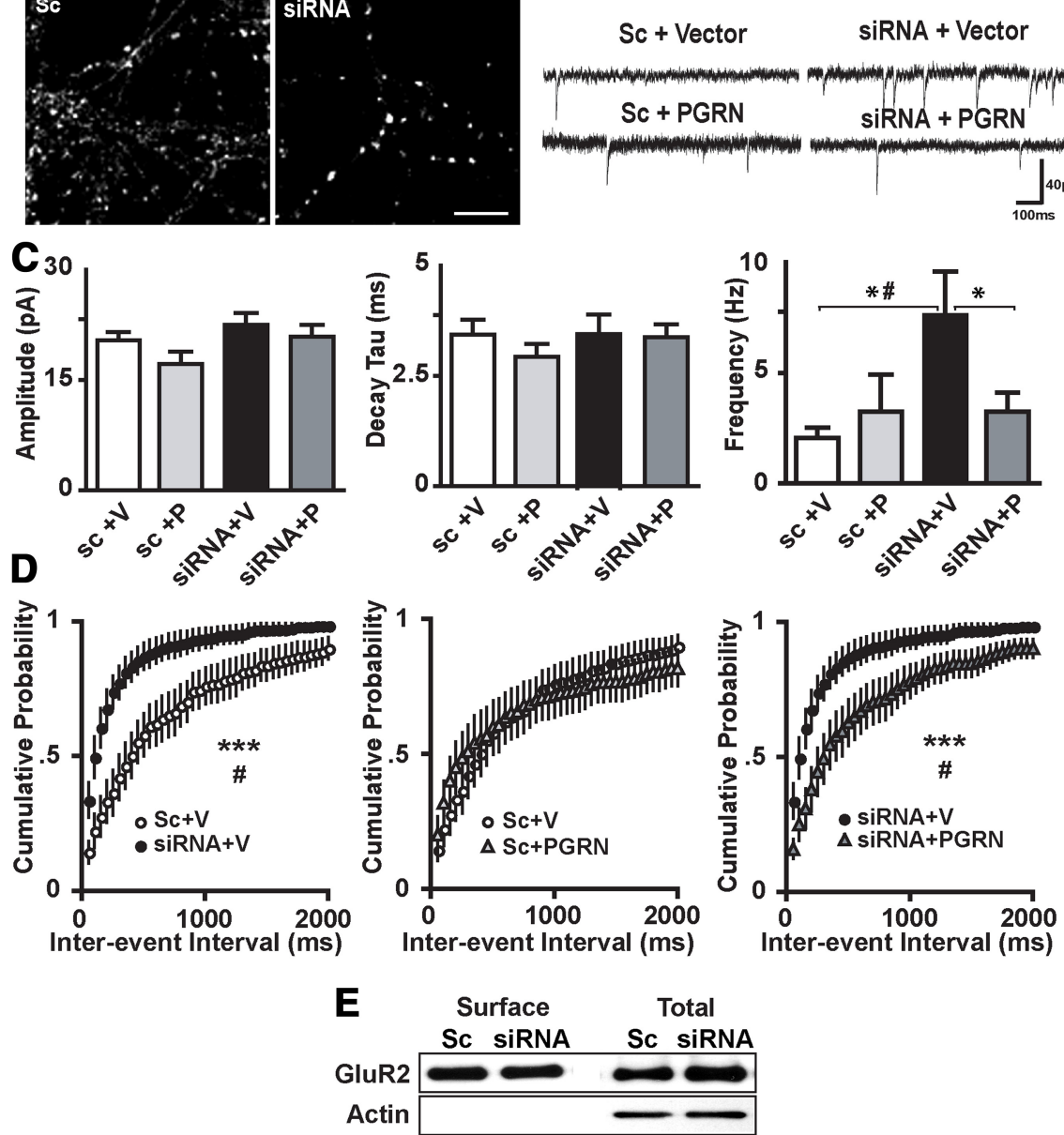

Figure 3. Synaptic vesicle recycling and the frequency of mEPSCs are enhanced in PGRN-deficient neurons. $A$, Representative images of FM4-64 staining of recycling synaptic vesicles in PGRN knockdown and control cultures. Scale bar, $10 \mu \mathrm{m}$. There was an increase in the integrated density of FM4-64 puncta (control: $100 \pm 8.6 \%$; siRNA: $128.0 \pm 3.7 \% ; p=0.04, n=3$ experiments). B, Representative traces showing mEPSCs. C, Mean mEPSC amplitudes (left) or decay kinetics (middle) are not significantly different in PGRN knockdown cultures; however, mean mEPSC frequency (right) is increased in PGRN knockdown cultures. $n=$ $8-13$ cells from 3-4 separate cultures; one-way Kruskal-Wallis ANOVA $p=0.024, \mathrm{KW}=9.46,{ }^{\#} p<0.05$ Dunn's post hoc, ${ }^{*} p<$ $0.05 t$ test direct comparison). $D$, Cumulative probability plots demonstrate that PGRN siRNA significantly reduces mEPSC IEls (left; two-way RM-ANOVA "treatment $p=0.0113, F_{(1,640)}=8.2$, ***interaction $\left.p=0.0001, F_{(40,640)}=6.6\right)$. Overexpression of PGRN does not significantly affect IEls (middle; $p=0.89$ ). The effect of siRNA on $m E P S C$ IEls is significantly reduced by subsequent PGRN CDNA expression (right; two-way RM-ANOVA ${ }^{\#}$ treatment $p=0.034, F_{(1,840)}=5.1$, ${ }^{* * *}$ interaction $p=0.0001, F_{(40,840)}=6.6$ ). $\boldsymbol{E}$, GluR2 levels are not significantly different in PGRN knockdown cultures. Surface GluR2: $1.26 \pm 0.22$-fold increase in surface GluR2 levels in PGRN siRNA cells compared to control ( $p=0.31$ ). Total GluR2: $1.08 \pm 0.26$-fold increase in total GluR2 levels in PGRN siRNA cells compared to control ( $p=0.78$ ). Shown is a representative blot from $n=3$ blots from 3 cultures. trol: $14.37 \pm 2.75 \%$; PGRN siRNA: $19.15 \pm$ $1.22 \% ; p=0.186, n=15$ images per condition, 3 cultures), nor were differences observed by the MTT assay for cell viability (control: $100 \pm 3.10 \%$; PGRN siRNA: $91.49 \pm 3.41 \%$; $p=0.076, n=4$ experiments, 4 cultures). Thus, PGRN protein levels can be reduced to $20 \%$ of normal expression in 2-week-old hippocampal neurons without significantly impacting survival.

To study the effect of PGRN knockdown on gross neuronal morphology, cells infected with scrambled and PGRN siRNAs were transiently transfected with a cDNA vector expressing the cell fill, DsRed. As a consequence of low transfection efficiency $(<1 \%)$, the morphology of individual neurons is clearly discerned and analyzed. There was a significant decrease in the number of primary neurites (Fig. $1 B, C$ ) and neurite length (Fig. 1D) in PGRN-deficient neurons compared to controls. This was rescued by reinfecting cultures with siRNA-insensitive PGRN, thereby indicating that these changes were not due to off-target effects (Fig. 1C,D).

Changes in neuronal morphology extended to modifications in the number and length of dendritic spines - the predominant site of excitatory postsynaptic connections. PGRN knockdown cells exhibited a reduced density of spine-like protrusions along dendrites, compared to controls (control: $100 \pm 3.19 \%$; siRNA: $69.17 \pm 5.27 \% ; p=3.6 \times 10^{-6}$ ) (Fig. $1 E, F)$. Moreover, there was a marked decrease in the density of mature, spine-like protrusions $(0-2 \mu \mathrm{m})$ and a significant increase in the density of longer "filopodial-like" protrusions (>11 $\mu \mathrm{m})$ (Fig. $1 F$ ).

We next examined the effects of PGRN knockdown on synapse density and size. Individual neurons were transfected at 11 DIV with the excitatory postsynaptic marker PSD-95 fused to red fluorescent a scrambled siRNA (control) or PGRN siRNA (PGRN knockdown). At 13 DIV (10 d after infection), PGRN levels were dramatically reduced in the siRNA-infected cells compared to controls (control: $100 \pm 6.92 \%$; siRNA: $21.38 \pm 6.85 \% p=$ $5.25 \times 10^{-5}, n=3$ blots from 3 cultures) (Fig. $1 A$ ). This $80 \%$ reduction in PGRN protein levels is comparable to the $65 \%$ decrease observed in the CSF of FTD patients with PGRN haploinsufficiency (Van Damme et al., 2008). To ensure that the phenotypic changes observed in cultures expressing PGRN siRNA were due to knockdown of PGRN specifically, PGRN was subsequently reintroduced to cells by reinfection of a construct expressing siRNA-insensitive, human PGRN (Fig. 1A).

Since PGRN has been shown to enhance neuronal survival (Van Damme et al., 2008), the effect of PGRN depletion on survival was determined at 13 DIV using TUNEL and MTT assays. There was no change in the percentage of total apoptotic nuclei in each field (con- protein (RFP) enabling the visualization of postsynaptic densities in individual cells. Synapses were defined by the colocalization of PSD-95-RFP and the presynaptic marker synaptophysin; however, it is important to note that these may not reflect functional, active synapses. There was a $37 \%$ reduction in the density of synapses in PGRN knockdown cultures compared to controls (control: $100 \pm 4.7 \%$; siRNA: $63.6 \pm 4.2 \%$; $p=1.3 \times 10^{-7}$ ) (Fig. $2 A, B)$.

To determine whether synaptic composition was also affected, cells were immunolabeled with antibodies against a number of presynaptic and postsynaptic proteins. Immunolabeling of cells for synaptophysin revealed a twofold increase in the size of synaptophysin puncta in PGRN knockdown cultures compared to controls (Fig. $2 B-D$ ). This was not due to increased expression of synaptophysin as assessed by Western blot (Fig. $2 E$ ). Changes in the ratio of functional excitatory/inhibitory synapses can lead to 

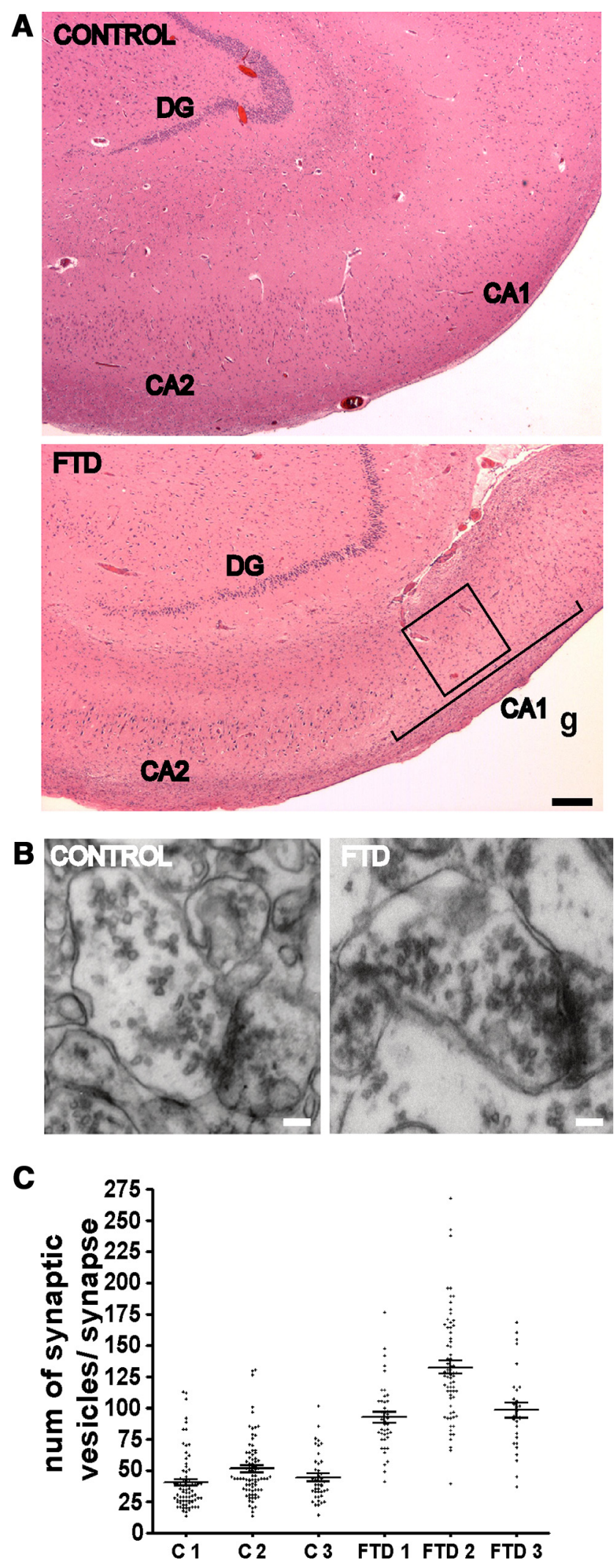

Figure 4. The number of synaptic vesicles per synapse is enhanced in the hippocampus of FTD patients with PGRN haploinsufficiency. $\boldsymbol{A}$, Representative hematoxylin and eosin staining of postmortem hippocampal sections from control and FTD with GRN mutations patients. The line demarcates the CA1 pyramidal cell layer that is virtually ablated in the FTD brain. The box approximates the region of stratum radiatum used for electron micrograph analyses. $\boldsymbol{B}$, Representative electron micrographs of hippocampal synapses in the stratum radiatum of control and FTD brains. $C$, The number of synaptic vesicles per synapse in FTD1-FTD3 $(107.83 \pm 12.50 ; n=$ neurodegeneration. To determine whether the increase in the size of presynaptic compartments is specific for excitatory or inhibitory synapses, cultures were immunolabeled with antibodies against VGlut-1 (a vesicular glutamate transporter localized at excitatory synapses) and VGAT (a vesicular GABA transporter localized at inhibitory synapses), respectively. The size of VGlut-1, and to a lesser extent VGAT puncta, was increased in PGRN knockdown cultures compared to controls (VGlut-1: control: $100 \pm 4.5 \%$; siRNA: $195.8 \pm 21.5 \% ; p=2.5 \times 10^{-4}, n=20-22$ images per condition, 2 cultures; VGAT: control: $100 \pm 5.1 \%$; siRNA: $131.8 \pm 10 \%$; $p=$ $0.007, n=30-33$ images per condition, 2 cultures). The size of postsynaptic PSD-95 clusters was unchanged (control: $100 \pm$ $2.6 \%$; siRNA: $88.1 \pm 5.1 \% ; p=0.038, n=30$ images per condition, 3 cultures)

The increase in the size of synaptophysin, VGAT, and VGlut-1 clusters suggests an increase in the number of synaptic vesicles per synapse. Using electron microscopy, we confirmed a $33 \%$ increase in the number of synaptic vesicles per excitatory asymmetric synapse in PGRN knockdown cultures (control: $63 \pm 2.8$; siRNA: $94 \pm 3.88 ; p=6.3 \times 10^{-3}, n=133$ and 146 synapses per condition, 3 cultures) (Fig. $2 F, G$ ). The length of the postsynaptic density was unchanged (control: $417 \pm 16 \mathrm{~nm}, n=110$ synapses; siRNA: $425 \pm 21 \mathrm{~nm} ; p=0.766, n=98$ synapses, 3 cultures). These data indicate that PGRN knockdown decreases dendritic arborization and synapse density. Although this suggests an overall reduction in neuronal connectivity, we also observed an increase in the number of synaptic vesicles per synapse, which may reflect an increase in transmission at remaining synapses. It is possible that increased synaptic vesicle number is a direct effect of decreased PGRN signaling, or a homeostatic response to decreased synapse number in the network.

To determine whether the increase in vesicles per synapse translates to changes in presynaptic function, vesicle turnover was assessed using the styryl dye FM4-64 (Betz and Bewick, 1992). The average integrated density (product of area and mean gray value) of FM4-64 clusters was increased in PGRN knockdown cultures (control: $100 \pm 8.6 \%$; siRNA: $128.0 \pm 3.7 \% ; p=0.04, n=20$ images per condition, 3 cultures), suggesting increased synaptic vesicle recycling at individual boutons.

To examine synaptic transmission in PGRN knockdown cultures compared to controls, whole-cell voltage-clamp recordings were conducted. Analysis of mEPSCs demonstrated that AMPA receptor-mediated current amplitudes and decay kinetics did not differ significantly across groups (Fig. $3 B, C$ ). In contrast, we observed a marked increase in the frequency of events in PGRN siRNA cultures (Fig. $3 B, C$, right). Due to the large number of events collapsed to a single cell mean, it is appropriate to consider the mean cell cumulative probability of mEPSC parameters between groups. There were no significant effects of PGRN siRNA or PGRN overexpression upon mEPSC amplitude cumulative probabilities; however, PGRN siRNA significantly decreased mEPSC interevent intervals (IEIs) (Fig. 3D, left), whereas PGRN overexpression had no effect (Fig. 3D, middle). Furthermore, in agreement with mean cell frequencies, subsequent PGRN overexpression abolished the siRNA-mediated decrease in mEPSC IEIs (Fig. 3D, right).

Together, the data demonstrate that reduced PGRN levels re-

143 synapses) was significantly higher than in control patients (C1-C $3 ; 45.53 \pm 3.2 ; n=199$ synapses; $p=0.031$, Student's $t$ test). Each datum represents the number of vesicles per synapse. Scale bars: $\boldsymbol{A}, 1 \mathrm{~mm} ; \boldsymbol{B}, 100 \mathrm{~nm}$ 
sult in an increased frequency of spontaneous glutamatergic transmission. This can be attributed to either of the following: (1) increased probability of transmitter release; or (2) an increase in the number of synapses with similar or reduced release probabilities. Given the reduction in overall synapse density in PGRN siRNA cultures (Fig. 2), the data suggest that presynaptic release probability is markedly increased at remaining synapses.

To further examine whether the increase in mEPSC frequency can be attributed to presynaptic or postsynaptic mechanisms, we examined the level of total and surface AMPA receptors in control and PGRN knockdown cultures. No differences were observed in either total or surface GluR2 (Fig. 3E) in agreement with a lack of change in mEPSC amplitude. Together, the data suggest that increased synaptic transmission is attributable to increased probability of presynaptic release.

We next determined whether the morphological changes observed in vitro were also observed in the brains of FTD patients with PGRN haploinsufficiency. Postmortem hippocampal sections were obtained from three control and three patients with familial FTD with GRN mutations (UBC-11, UBC-15, and UBC-17) exhibiting severe FTD and a marked loss of CA1 hippocampal pyramidal neurons (Fig. 4A). Electron microscopy analysis demonstrated a significant increase in the number of synaptic vesicles per synapse in FTD patients compared to control patients (Fig. $4 B, C$ ).

\section{Discussion}

Although dementia is typically thought of as a disease caused by the process of aging, emerging data indicate that there may be a previously unrecognized neurodevelopmental component to the occurrence of FTD. Indeed, comparisons of family members with and without pathogenic mutations leading to FTD demonstrate that presymptomatic carriers exhibit cognitive dysfunction many decades before the predicted onset of dementia (Geschwind et al., 2001). Understanding the normal function of proteins involved in the etiology of FTD and how they impact not only neurodegeneration but also neural connectivity and function is, therefore, of the utmost importance.

Our data provide insight into of the normal functioning of PGRN in the early development and maintenance of neural connectivity. As PGRN is widely expressed in both neurons and glia during development, it would be of interest to determine the contribution of glial-derived PGRN in brain development. Furthermore, PGRN is highly expressed in specific neuronal populations throughout adulthood, and further work is required to determine whether PGRN plays a role in the maintenance of synaptic connections.

It is clear that when considering the etiology of FTD, other factors beyond PGRN haploinsufficiency must be taken into consideration. For example, FTD patients with PGRN haploinsufficiency also exhibit ubiquitin-positive, TDP-43-positive neuronal inclusions that are believed to be associated with the pathophysiology of FTD. Patients also exhibit pronounced atrophy of specific brain regions, significant astrocytic gliosis, and myelin loss in white matter tracts. More specifically, in the hippocampus, there is a marked loss of neurons and gliosis in area CA1 and the subiculum (for review, see Eriksen and Mackenzie, 2008). It is intriguing that depletion of PGRN in both hippocampal cultures and patients with FTD results in increased number of vesicles per synapse, yet it is highly probable that neuronal inclusions, gliosis, and myelin loss also exert significant effects on neural function in patients with severe FTD. Further investigations will determine whether prolonged PGRN-haploinsufficiency and the associated neuronal dysfunction reported here are related, or even causal to, latent pathophysiological processes in FTD.

\section{References}

Baker CA, Manuelidis L (2003) Unique inflammatory RNA profiles of microglia in Creutzfeldt-Jakob disease. Proc Natl Acad Sci USA 100:675-679.

Baker M, Mackenzie IR, Pickering-Brown SM, Gass J, Rademakers R, Lindholm C, Snowden J, Adamson J, Sadovnick AD, Rollinson S, Cannon A, Dwosh E, Neary D, Melquist S, Richardson A, Dickson D, Berger Z, Eriksen J, Robinson T, Zehr C, et al. (2006) Mutations in progranulin cause tau-negative frontotemporal dementia linked to chromosome 17. Nature 442:916-919.

Betz WJ, Bewick GS (1992) Optical analysis of synaptic vesicle recycling at the frog neuromuscular junction. Science 255:200-203.

Bronner IF, Rizzu P, Seelaar H, van Mil SE, Anar B, Azmani A, Donker Kaat L, Rosso S, Heutink P, van Swieten JC (2007) Progranulin mutations in Dutch familial frontotemporal lobar degeneration. Eur J Hum Genet 15:369-374.

Cruts M, Gijselinck I, van der Zee J, Engelborghs S, Wils H, Pirici D, Rademakers R, Vandenberghe R, Dermaut B, Martin JJ, van Duijn C, Peeters K, Sciot R, Santens P, De Pooter T, Mattheijssens M, Van den Broeck M, Cuijt I, Vennekens K, De Deyn PP, et al. (2006) Null mutations in progranulin cause ubiquitin-positive frontotemporal dementia linked to chromosome 17q21. Nature 442:920-924.

Daniel R, He Z, Carmichael KP, Halper J, Bateman A (2000) Cellular localization of gene expression for progranulin. J Histochem Cytochem 48:999-1009.

Daniel R, Daniels E, He Z, Bateman A (2003) Progranulin (acrogranin/PC cell-derived growth factor/granulin-epithelin precursor) is expressed in the placenta, epidermis, microvasculature, and brain during murine development. Dev Dyn 227:593-599.

Eriksen JL, Mackenzie IR (2008) Progranulin: normal function and role in neurodegeneration. J Neurochem 104:287-297.

Gao X, Joselin AP, Wang L, Kar A, Ray P, Bateman A, Goate AM, Wu JY (2010) Progranulin promotes neurite outgrowth and neuronal differentiation by regulating GSK-3beta. Protein Cell 1:552-562.

Gass J, Cannon A, Mackenzie IR, Boeve B, Baker M, Adamson J, Crook R, Melquist S, Kuntz K, Petersen R, Josephs K, Pickering-Brown SM, GraffRadford N, Uitti R, Dickson D, Wszolek Z, Gonzalez J, Beach TG, Bigio E, Johnson N, et al. (2006) Mutations in progranulin are a major cause of ubiquitin-positive frontotemporal lobar degeneration. Hum Mol Genet 15:2988-3001.

Geschwind DH, Robidoux J, Alarcón M, Miller BL, Wilhelmsen KC, Cummings JL, Nasreddine ZS (2001) Dementia and neurodevelopmental predisposition: cognitive dysfunction in presymptomatic subjects precedes dementia by decades in frontotemporal dementia. Ann Neurol 50:741-746.

Holman D, Henley JM (2007) A novel method for monitoring the cell surface expression of heteromeric protein complexes in dispersed neurons and acute hippocampal slices. J Neurosci Methods 160:302-308.

Kleinberger G, Wils H, Ponsaerts P, Joris G, Timmermans JP, Van Broeckhoven C, Kumar-Singh S (2010) Increased caspase activation and decreased TDP-43 solubility in progranulin knockout cortical cultures. J Neurochem 115:735-747.

Korting HC, Schindler S, Hartinger A, Kerscher M, Angerpointner T, Maibach HI (1994) MTT-assay and neutral red release (NRR)-assay: relative role in the prediction of the irritancy potential of surfactants. Life Sci 55:533-540.

Malaspina A, Kaushik N, de Belleroche J (2001) Differential expression of 14 genes in amyotrophic lateral sclerosis spinal cord detected using gridded cDNA arrays. J Neurochem 77:132-145.

Milnerwood AJ, Raymond LA (2007) Corticostriatal synaptic function in mouse models of Huntington's disease: early effects of huntingtin repeat length and protein load. J Physiol 585:817-831.

Milnerwood AJ, Gladding CM, Pouladi MA, Kaufman AM, Hines RM, Boyd JD, Ko RW, Vasuta OC, Graham RK, Hayden MR, Murphy TH, Raymond LA (2010) Early increase in extrasynaptic NMDA receptor signaling and expression contributes to phenotype onset in Huntington's disease mice. Neuron 65:178-190.

Mukherjee O, Pastor P, Cairns NJ, Chakraverty S, Kauwe JS, Shears S, Behrens 
MI, Budde J, Hinrichs AL, Norton J, Levitch D, Taylor-Reinwald L, Gitcho M, Tu PH, Tenenholz Grinberg L, Liscic RM, Armendariz J, Morris JC, Goate AM (2006) HDDD2 is a familial frontotemporal lobar degeneration with ubiquitin-positive, tau-negative inclusions caused by a missense mutation in the signal peptide of progranulin. Ann Neurol 60:314-322.

Petkau TL, Neal SJ, Orban PC, MacDonald JL, Hill AM, Lu G, Feldman HH, Mackenzie IR, Leavitt BR (2010) Progranulin expression in the developing and adult murine brain. J Comp Neurol 518:3931-3947.

Pickering-Brown SM, Baker M, Gass J, Boeve BF, Loy CT, Brooks WS, Mackenzie IR, Martins RN, Kwok JB, Halliday GM, Kril J, Schofield PR, Mann DM, Hutton M (2006) Mutations in progranulin explain atypical phenotypes with variants in MAPT. Brain 129:3124-3126.

Ryan CL, Baranowski DC, Chitramuthu BP, Malik S, Li Z, Cao M, Minotti S, Durham HD, Kay DG, Shaw CA, Bennett HP, Bateman A (2009) Progranulin is expressed within motor neurons and promotes neuronal cell survival. BMC Neurosci 10:130.
Sun Y, Aiga M, Yoshida E, Humbert PO, Bamji SX (2009) Scribble interacts with beta-catenin to localize synaptic vesicles to synapses. Mol Biol Cell 20:3390-3400.

Van Damme P, Van Hoecke A, Lambrechts D, Vanacker P, Bogaert E, van Swieten J, Carmeliet P, Van Den Bosch L, Robberecht W (2008) Progranulin functions as a neurotrophic factor to regulate neurite outgrowth and enhance neuronal survival. J Cell Biol 181:37-41.

van der Zee J, Le Ber I, Maurer-Stroh S, Engelborghs S, Gijselinck I, Camuzat A, Brouwers N, Vandenberghe R, Sleegers K, Hannequin D, Dermaut B, Schymkowitz J, Campion D, Santens P, Martin JJ, Lacomblez L, De Pooter T, Peeters K, Mattheijssens M, Vercelletto M, et al. (2007) Mutations other than null mutations producing a pathogenic loss of progranulin in frontotemporal dementia. Hum Mutat 28: 416.

Xie C, Markesbery WR, Lovell MA (2000) Survival of hippocampal and cortical neurons in a mixture of MEM + and B27-supplemented neurobasal medium. Free Radic Biol Med 28:665-672. 